\title{
BMJ Open Rationale and design of a prospective substudy of clinical endpoint adjudication processes within an investigator-reported randomised controlled trial in patients with coronary artery disease: the GLOBAL LEADERS Adjudication Sub-StudY (GLASSY)
}

To cite: Leonardi S, Franzone A, Piccolo R, et al. Rationale and design of a prospective substudy of clinical endpoint adjudication processes within an investigator-reported randomised controlled trial in patients with coronary artery disease: the GLOBAL LEADERS Adjudication SubStudY (GLASSY). BMJ Open 2019;9:e026053. doi:10.1136/ bmjopen-2018-026053

- Prepublication history and additional material for this paper are available online. To view these files, please visit the journal online (http://dx.doi. org/10.1136/bmjopen-2018026053).

$\mathrm{SL}$ and $\mathrm{AF}$ contributed equally.

Received 19 August 2018 Revised 20 November 2018 Accepted 21 January 2019

Check for updates

(C) Author(s) (or their employer(s)) 2019. Re-use permitted under CC BY-NC. No commercial re-use. See rights and permissions. Published by BMJ.

For numbered affiliations see end of article.

Correspondence to Dr Marco Valgimigli; marco.valgimigli@insel.ch

Sergio Leonardi, ${ }^{1}$ Anna Franzone, ${ }^{2}$ Raffaele Piccolo, ${ }^{2}$ Eugene McFadden, ${ }^{3}$ Pascal Vranckx, ${ }^{4}$ Patrick Serruys, ${ }^{5}$ Edouard Benit, ${ }^{6}$ Christoph Liebetrau, ${ }^{7}$ Luc Janssens, ${ }^{8}$ Maurizio Ferrario, ${ }^{9}$ Aleksander Zurakowski, ${ }^{10}$

Robert-Jan van Geuns, ${ }^{11}$ Marcello Dominici, ${ }^{12}$ Kurt Huber, ${ }^{13}$ Ton Slagboom, ${ }^{14}$ Paweł Buszman, ${ }^{15}$ Leonardo Bolognese, ${ }^{16}$ Carlo Tumscitz, ${ }^{17}$ Krzysztof Bryniarski, ${ }^{18}$ Adel Aminian, ${ }^{19}$ Mathias Vrolix, ${ }^{20}$ Ivo Petrov, ${ }^{21}$ Scot Garg, ${ }^{22}$ Christoph Naber, ${ }^{23}$ Janusz Prokopczuk, ${ }^{24}$ Christian Hamm, ${ }^{25}$ Gabriel Steg, ${ }^{26}$ Dik Heg, ${ }^{27}$ Peter Juni, ${ }^{28}$ Stephan Windecker, ${ }^{29}$ Marco Valgimigli $^{29}$

\section{ABSTRACT}

Introduction The GLOBAL LEADERS is an open-label, pragmatic and superiority randomised controlled trial designed to challenge the current treatment paradigm of dual antiplatelet therapy (DAPT) for 12 months followed by aspirin monotherapy among patients undergoing percutaneous coronary intervention. By design, all study endpoints are investigator reported (IR) and not subject to formal adjudication by an independent Clinical Event Committee (CEC), which may introduce detection, reporting or ascertainment bias.

Methods and analysis We designed the GLOBAL LEADERS Adjudication Sub-StudY (GLASSY) to prospectively implement, in a large sample of patients enrolled within the GLOBAL LEADERS trial (7585 of $15991,47.5 \%)$, an independent adjudication process of reported and unreported potential endpoints, using standardised CEC procedures, in order to assess whether 23-month ticagrelor monotherapy (90 mg twice daily) after 1-month DAPT is non-inferior to a standard regimen of DAPT for 12 months followed by aspirin monotherapy for the primary efficacy endpoint of death, nonfatal myocardial infarction, non-fatal stroke or urgent target vessel revascularisation and superior for the primary safety endpoint of type 3 or 5 bleeding according to the Bleeding Academic Research Consortium criteria. This study will comprehensively assess the comparative safety and efficacy of the two tested antithrombotic strategies on CEC-adjudicated ischaemic and bleeding endpoints and will provide insights into the role of a standardised CEC adjudication process on the interpretation of study findings by quantifying the level of concordance between IR-reported and CEC-adjudicated events.

\section{Strengths and limitations of this study}

- GLOBAL LEADERS Adjudication Sub-StudY (GLASSY) is a comprehensive, rigorous and standardised assessment of several non-fatal endpoints as well as death (including type, mechanism and relationship to bleeding) in a representative sample of the GLOBAL LEADERS trial performed according to best practices of adjudication.

- An intrinsic limitation is that GLOBAL LEADERS has been designed as an investigator-reported (IR)-only study. Therefore, systematic identification of study endpoints is limited by the electronic case report form (eCRF) and relies on source documentation provided by the site, which reduces the ability to identify all possible potential endpoints.

- For feasibility, GLASSY will be conducted in a sample rather than the entire parent study, which may bias the study towards the null hypothesis of no difference between IR-adjudicated and Clinical Event Committee-adjudicated endpoint by selecting best enrolling sites. While this bias is possible, the relatively large study sample ( $\approx 50 \%$ of the parent study) makes this possibility unlikely.

Ethics and dissemination GLASSY has been approved by local ethics committee of all study sites and/or by the central ethics committee for the country depending on country-specific regulations. In all cases, they deemed that it was not necessary to obtain further informed consent from individual subjects.

Trial registration number NCT01813435. 


\section{RATIONALE}

The prolonged combination of aspirin and a $\mathrm{P}_{2} \mathrm{Y}_{12}$ receptor inhibitor, typically for 12 months, represents the established antiplatelet therapy in patients with or without acute coronary syndrome (ACS) undergoing percutaneous coronary intervention (PCI) with drugeluting stent implantation. ${ }^{1}$ Clopidogrel, an inconsistent $\mathrm{P}_{2} \mathrm{Y}_{12}$ receptor inhibitor ${ }^{2}$ with considerable variability in interpatient response, ${ }^{3}$ proved inferior to stronger and more consistent $\mathrm{P}_{2} \mathrm{Y}_{12}$ inhibitors, such as ticagrelor, in preventing ischaemic and thrombotic cardiovascular events among patients with ACS. ${ }^{4}$ With the introduction and widespread adoption in clinical practice of more potent $\mathrm{P}_{2} \mathrm{Y}_{12}$ inhibitors, it has been hypothesised that the addition of aspirin may yield little additional inhibition of platelet aggregation and marginal incremental clinical benefit compared with a strategy based on potent P2Y ${ }_{12}$ receptor inhibitor monotherapy. ${ }^{56}$ This led to the hypothesis that ticagrelor monotherapy may have similar efficacy compared with the combination of aspirin and ticagrelor and be better tolerated.

The GLOBAL LEADERS trial was designed to challenge the current treatment paradigm consisting of 12-month dual antiplatelet therapy (DAPT; clopidogrel+aspirin among patients with stable coronary artery disease (CAD); ticagrelor+aspirin among patients with ACS) followed by aspirin monotherapy in patients undergoing PCI based on the superiority for the composite endpoint of all-cause death or Q-wave myocardial infarction (MI) assessed at 2 years. ${ }^{7}$ It is an open-label, randomised comparison testing an innovative antithrombotic regimen of 23-month ticagrelor $90 \mathrm{mg}$ twice daily monotherapy after 1-month DAPT (ticagrelor $90 \mathrm{mg}$ twice daily plus low-dose aspirin) against conventional 12-month DAPT in all-comer patients undergoing PCI with bivalirudin-supported, biolimus-eluting stent implantation. The GLOBAL LEADERS is a pragmatic clinical trial, and by design, all study endpoints are investigator reported (IR) and therefore not adjudicated by an independent Clinical Event Committee (CEC). Only new Q-wave MI will be identified by independent core lab assessment and validated by a physician blinded to treatment allocation. All other endpoints, including specific causes of mortality, non-Qwave MI, stroke, stent thrombosis and bleeding will be analysed as reported by the local investigators.

Although the use of IR endpoints in a phase III randomised trial is a simple and less expensive alternative, their sole use has potential to introduce detection, reporting or ascertainment bias, especially in the absence of blinding to randomised treatment (ie, in an open-label design as in the case of the GLOBAL LEADERS trial). This might challenge the interpretation of the GLOBAL LEADERS study results, especially as it relates to the effect of the randomly allocated treatment on non-fatal clinical endpoints. Moreover, the design of GLOBAL LEADERS also raises important questions regarding bleeding adverse events that may differ between groups.
We, therefore, designed the GLOBAL LEADERS Adjudication Sub-StudY (GLASSY) with the aim to prospectively implement, in a representative sample of patients enrolled within the GLOBAL LEADERS trial, an independent adjudication process of reported as well as unreported potential endpoints, leveraging on standardised CEC procedures. This GLASSY substudy is powered to test whether 23-month ticagrelor monotherapy after a short course of DAPT for 1 month is non-inferior to conventional 12-month DAPT followed by aspirin monotherapy with respect to CEC-adjudicated death, non-fatal MI, non-fatal stroke or urgent target vessel revascularisation (TVR) and superior in preventing CEC-adjudicated major bleeding. Furthermore, GLASSY will evaluate the implications of the CEC adjudication process for the interpretation of study results by quantifying the level of concordance between IR-reported and CEC-adjudicated events and will define the role of CEC adjudication process for the assessment of the efficacy and safety of the randomised antithrombotic strategies on a broader set of fatal and non-fatal clinical endpoints.

\section{DESIGN}

\section{Parent study}

The GLOBAL LEADERS study is a superiority, openlabel, multicentre, randomised controlled trial (RCT) in an all-comer population of patients, presenting with ACS or stable coronary artery disease, undergoing PCI with the uniform use of Biolimus A9-eluting stents (BioMatrix BES; Biosensors Europe SA, Morges, Switzerland) and receiving bivalirudin at the time of the index procedure (figure 1). A total of 15991 patients have been randomly assigned 1:1 to ticagrelor $90 \mathrm{mg}$ twice daily for 24 months plus aspirin $\leq 100 \mathrm{mg}$ daily for 1 month (experimental arm) or standard DAPT with either ticagrelor, in case of ACS, or clopidogrel, in case of stable coronary artery disease, for 12 months plus aspirin $\leq 100 \mathrm{mg}$ daily for 24 months (control arm). All study endpoints are IR with randomisation stratified by enrolling site as well as clinical presentation. The primary endpoint of the GLOBAL LEADERS is the composite of all-cause death or new $\mathrm{Q}$-wave MI at 24 months. The presence and date of new Q-wave MI will be identified by an independent ECG core laboratory and validated by a single physician blinded to treatment allocation using adverse events reported in the electronic case report form (eCRF) supplemented, if required, by additional source documents. The key safety endpoint is IR class 3 or 5 bleeding according to the Bleeding Academic Research Consortium (BARC) definitions. Other secondary endpoints include stroke, MI, coronary revascularisation and definite stent thrombosis. As pragmatic trial, GLOBAL LEADERS implemented a risk-based monitoring process for site-based operational activities favouring centralised remote monitoring rather than in-person on-site monitoring. GLOBAL LEADERS terminated enrolment on 9 November 2015. 


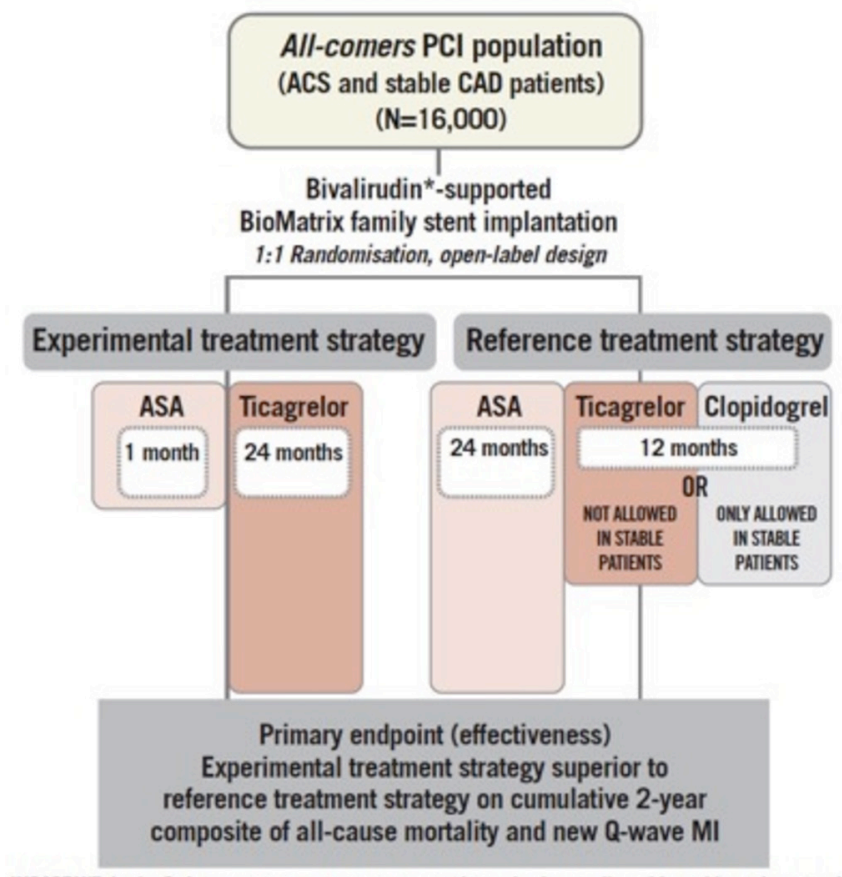

IMPORTANT: In the Reference treatment strategy arm, ticagrelor is not allowed in stable patients, and clopidogrel must be given in combination with ASA. However, patients already on stable maintenance treatment with ticagrelor (or prasugrel) can continue with ticagrelor treatment (for 12 months post index $\mathrm{PCI}$ ).

"In countries where available

Figure 1 GLOBAL LEADERS design. ACS, acute coronary syndrome; ASA, aspirin; CAD, coronary artery disease; $\mathrm{Ml}$, myocardial infarction; $\mathrm{PCl}$, percutaneous coronary intervention.

\section{Objectives}

The primary objective of the GLASSY is to assess, in a representative subgroup of patients enrolled within the
GLOBAL LEADERS study, whether 23-month ticagrelor monotherapy after a short course of DAPT (1 month) is non-inferior to conventional 12-month DAPT followed by aspirin monotherapy for the composite endpoint of CEC-adjudicated all-cause death, non-fatal MI, non-fatal stroke or urgent TVR, and superior in preventing CEC-adjudicated major bleeding (BARC types 3 and 5 ) in an all-comers population undergoing PCI at 24 months (figure 2). A secondary objective is to quantify the level of concordance between IR-adjudicated and CEC-adjudicated endpoints.

\section{Endpoints}

GLASSY will have two independent, CEC-adjudicated, coprimary endpoints at 24 months:

1. The composite of death, non-fatal MI, non-fatal stroke or urgent TVR (coprimary efficacy endpoint).

2. The composite of BARC type 3 or 5 bleeding (coprimary safety endpoint).

Secondary endpoints will include:

- Each component of the coprimary composite endpoints.

- Definite, probable or possible stent thrombosis according to Academic Resaerch Consortium (ARC) classification.

- Bleeding events according to BARC (primary safety endpoint) as well as the alternative Thrombolysis In Myocardial Infarction (TIMI) and Global Utilization of Streptokinase and Tissue Plasminogen Activator for Occluded Coronary Arteries (GUSTO) classifications.

- Type of death (cardiovascular vs non-cardiovascular and subtypes).

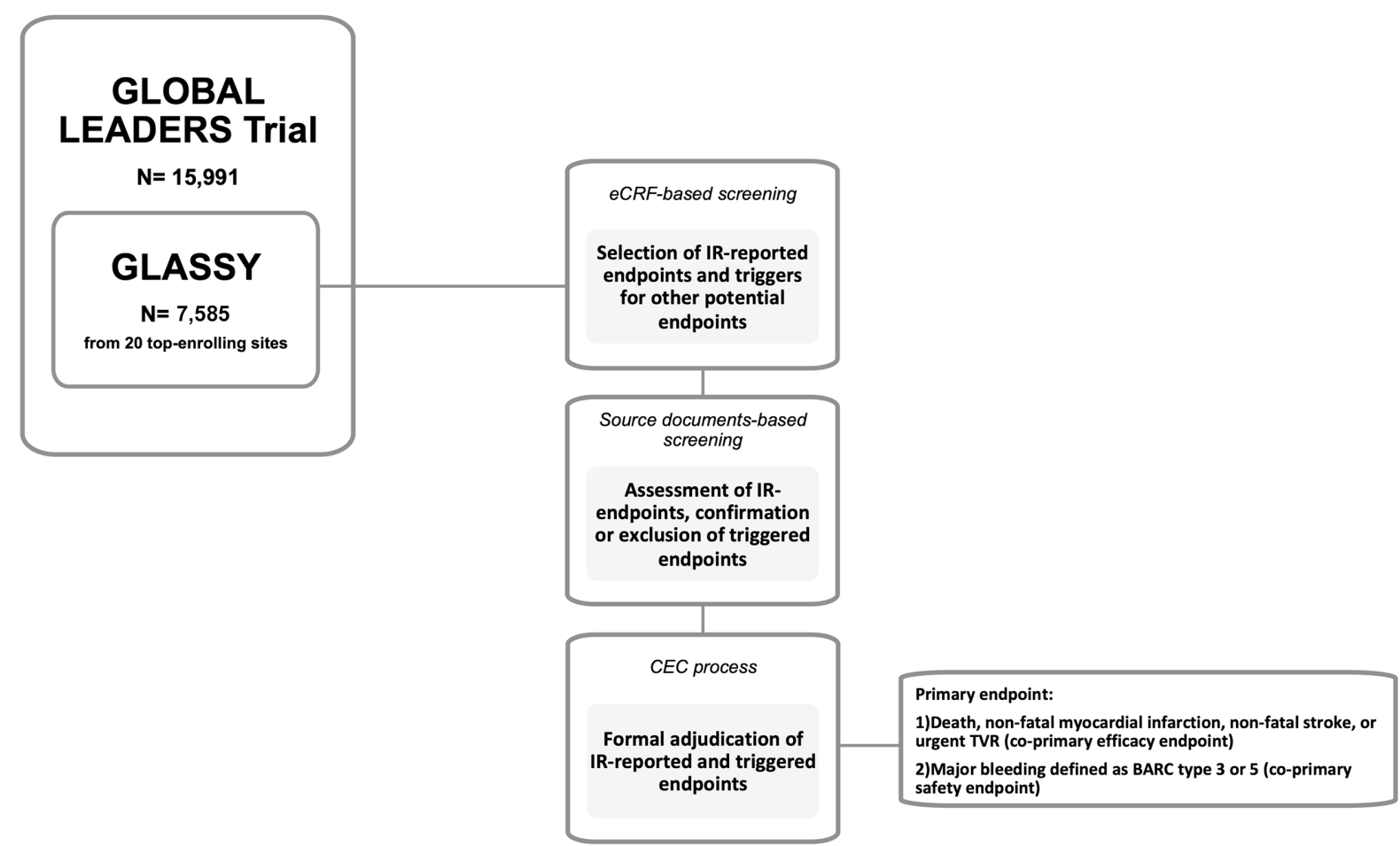

Figure 2 GLASSY design. CEC, Clinical Event Committee; GLASSY, GLOBAL LEADERS Adjudication Sub-StudY. 


\section{CEC procedures}

According to best adjudication practice, ${ }^{8}$ GLASSY is being conducted according to the following features:

\section{Prospective approach to adjudication}

The CEC dataset will be locked before the termination of the parent study. Suspected events (triggers) will be assessed during the conduct of the study rather than adjudicating all cases after the study is completed and the primary results are available (ie, retrospective adjudication). In case of updated entry of suspected events or updated source documentation by the site after request by the CEC team of source documentation, events will be re-evaluated for adjudication.

\section{Blinding of randomised treatment allocation}

According to the prospective randomized open blinded end-point (PROBE) methodology, ${ }^{9} 10$ the CEC will be blinded to randomised treatment allocation.

Several steps will be undertaken to ensure that the CEC personnel and physicians remain blinded.

First, any reference to treatment assignment contained in the eCRF or source documents that could lead to unblinding of treatment assignment will be obliterated by using a black marker by the site prior to submission to the CEC physician members.

Second, the CEC coordinator and operation personnel will obliterate any reference to study drug assignment prior to distribution to the physicians if information is noted during the preparation of the event packet.

Third, if a reviewer notes the treatment assignment during the review of a particular event, the CEC coordinator is notified, and the event is sent for review by the third expert reviewer.

Triggering and adjudication of IR as well as non-IR events

All IR events (death, MI, stroke, bleeding, coronary revascularisation and stent thrombosis) will be adjudicated by the CEC through dedicated case report forms (CRFs) (online online supplementary appendix). We will also use comprehensive search strategies for potential cardiovascular events that are not reported by the investigator via eCRF dedicated queries. Indeed, it may happen that patients without IR events or triggers may have experienced an event qualifying for the endpoints of the GLASSY study. ${ }^{10}$

It is possible that the request of source documentation may trigger endpoint reporting (and bias the study towards the null hypothesis). To quantify this, IR endpoints entered after CEC requested source documentation will be monitored and reported.

\section{Independent voting processes}

Independent voting processes by CEC members with at least 3 CEC members (see online supplementary appendix A) with knowledge of the geographic variations of care represented in the trial. Each event will be reviewed independently by at least two CEC physicians. In case of disagreement, the event will be reviewed by a
Committee of at least three reviewers with independent vote.

\section{Independence from parent study}

To maximise the scientific integrity of GLASSY, CEC personnel will operate independently from the data management group of the parent study, including no crosstalk on trigger logic specifications, query processes for source documentation and most importantly event reporting and adjudication results.

\section{Quantification of sufficient evidence for adjudication of non-fatal} triggers (no vs unknown events)

Finally, we will quantify the minimum amount of evidence required for the assessment of non-fatal endpoints. In a randomised trial, a prerequisite to assess whether a suspected non-fatal endpoint has occurred or not is the availability of sufficient evidence for such an assessment, including relevant source documents, tests and/or laboratory exams. While this is commonly performed for fatal events (death is adjudicated as 'unknown' in case of no or insufficient description of death circumstances), it is not generally mandatory for non-fatal events.

In GLASSY, we will report all non-fatal endpoints, but for each non-fatal trigger examined, an assessment will be performed as to whether enough information is available for formal adjudication. This will allow distinguishing triggers that did not meet the endpoint definition (ie, no event with sufficient documentation present) from triggers for which this is unknown due to insufficient documentation. For each type of non-fatal endpoint, the proportion of events with insufficient evidence will indirectly estimate: (A) the feasibility of GLASSY; (B) the quality of endpoint reported by sites; and $(\mathrm{C})$ the uncertainty of the evidence related to the studied outcome.

Sufficient evidence for CEC adjudication includes at a minimum a narrative description with at least one pertinent medical documentation, including ECG/biomarkers for MI; angiographic report for stent thrombosis and urgent revascularisation; brain imaging for stroke; and labs and other appropriate testing for bleeding. In case of CRF-only narrative, the evidence will be considered insufficient, and the case will not undergo CEC adjudication.

\section{Quality control of the adjudication process}

To ensure the highest reproducibility, a random sample of $\approx 5 \%$ of adjudicated events will be rereviewed by the complete CEC committee (ie, three members) who are blinded to the initial results.

A major disagreement will be considered if there was a disagreement on whether an event had occurred, while a minor disagreement is any discordance on the remaining adjudicated fields. Major disagreement will be reported as part of the final study report and will be used to identify the presence of systematic problems in the adjudication process. 


\section{CEC operations}

Within the selected study patients, all IR events as well as additional potential events (triggers) identified through a systematic analysis of the eCRF form will be considered for CEC adjudication. Non-IR triggers will be assessed after all the relevant source documentation has been requested to and provided by the participating sites and will be identified using a comprehensive search strategy that consider keywords logically related to the event. In general, key words with a clear relationship to the endpoint of interest (eg, for MI: unstable angina or ischaemic heart disease) will trigger a formal CEC review, whereas keywords with a potential relationship (eg, for MI: asystole, cardiac tamponade and hypertensive crisis) will trigger a review by a physician (independent from the CEC members) (online supplementary appendix). In the latter case, the event will undergo formal CEC review only if the reviewing physician will suspect an event. To limit possible reporting bias towards the null hypothesis (ie, querying for source documentation may stimulate a site to report previously unreported endpoints), only patients who have successfully completed the follow-up, data entry and all query processes for the parent study will be deemed eligible for the GLASSY study. For sites whose first language is not English, a mother tongue medical doctor will be involved for source documentation translation.

The first approval for GLASSY occurred on 18 April 2017, and the first adjudication has been performed on 6 September 2017.

\section{STATISTICAL ANALYSES AND SAMPLE SIZE CONSIDERATIONS}

The coprimary efficacy endpoint will be first tested as non-inferiority followed by a superiority testing only if non-inferiority criteria will be met. As the experimental treatment is simpler than the control treatment, it may be useful in patients with low drug adherence and/or who become intolerant to aspirin. For this reason, GLASSY adopted a non-inferiority design for one of the two coprimary endpoints. The coprimary safety endpoint will be tested with a superiority hypothesis only. Alpha error will be evenly split (2.5\% each) between the two coprimary endpoints. Based on best available data at the time of study design, the expected rate of the coprimary efficacy composite endpoint of death, non-fatal MI, non-fatal stroke or urgent TVR is $11 \%$ at 24 months in the control group. The expected rate of coprimary safety endpoint of type 3 or 5 BARC bleeding is $5 \%$ at 24 months in the control group. For the coprimary efficacy endpoint, non-inferiority will be declared if the upper limit of the 95\% CI for the experimental (ie, ticagrelor monotherapy) versus conventional arm at 24 months is less than 1.22 on a risk ratio scale, corresponding to $2.2 \%$ absolute risk difference. A total of 3340 patients per group (6680 patients) will yield $85 \%$ power to detect non-inferiority with a one-sided type I error (alpha) of $2.5 \%$. The risk ratio will be calculated using the Mantel-Cox logrank method.

If non-inferiority will be met, 3593 patients per group (7186 patients) will provide $80 \%$ power to assess the superiority for the coprimary efficacy endpoint at 24 months, assuming $20 \%$ relative risk reduction in the experimental arm and a two-sided alpha of $2.5 \%$. A total of 7186 patients will provide more than $80 \%$ power to detect a relative risk reduction of $33 \%$ in the experimental arm at 2 years with respect to coprimary safety endpoint of BARC 3 or 5 bleeding, setting the two-sided alpha error at $2.5 \%$. For each trigger, the CEC-adjudicated events will be used if the evidence is sufficient and the IR endpoint if the evidence is not sufficient (ie, 'best available' data).

\section{Representativeness of the selected study cohort}

There is no a priori attempt to select a patient population in GLASSY that could be entirely representative of the whole population included in the parent study. This would require random selection of the sample at the patient level or at least at the site level which, although ideally desirable, would be financially unsustainable for an investigator-initiated study.

Importantly, in GLOBAL LEADERS, the randomisation was stratified by site. This means that GLASSY is a randomised substudy of the parent study, and therefore, the estimation of treatment effects are expected to be valid.

Baseline characteristics, quality indicators and risk profile of GLOBAL LEADERS patients according to GLASSY inclusion are presented in tables 1 and 2, with no significant interactions on any of the variables considered.

The estimated minimum sample size was 7186 . Therefore, to minimise the number of participating sites, only those with the highest recruitment rate based on the final number of included subjects were included. Accordingly, the top 19 recruiting sites would have provided an overall of 7365 patients. These 19 top-ranking recruitment sites were invited in Q1 2017 and all agreed to participate.

Local and, where deemed necessary, central institutional review approval was sought for all 19 participating sites in the form of either a protocol addendum or site-specific amendment. In Q1 2018, due to delays in getting study approved for the Bulgarian site ranked at 19th position, the invitation to participate was extended to an additional site that was ranked at 20th position. This would allow reaching a final population of 7585 patients.

\section{Evaluation of the concordance between IR-adjudicated and CEC-adjudicated endpoints}

Concordance between IR-adjudicated and CEC-adjudicated endpoints will be assessed in events with sufficient evidence only. We will use the Cohen's kappa with exact binomial $95 \%$ CIs as a measurement of the extent of agreement beyond chance alone. Cohen originally suggested the Kappa result be interpreted as follows: values $\leq 0$ as indicating no agreement and $0.01-0.20$ as none to slight, $0.21-0.40$ as fair, $0.41-0.60$ as moderate, 
Table 1 Baseline characteristics of GLOBAL LEADERS patients according to GLASSY inclusion

\begin{tabular}{|c|c|c|c|}
\hline & \multirow{2}{*}{$\begin{array}{l}\text { GLASSY } \\
\text { (20 sites) } \\
\mathrm{n}=7585 \\
\end{array}$} & \multirow{2}{*}{$\begin{array}{l}\text { No GLASSY } \\
\text { (110 sites) } \\
n=8383\end{array}$} & \multirow[b]{2}{*}{ P value } \\
\hline & & & \\
\hline Female & $n=7585,1799(23.7 \%)$ & $n=8383,1915(22.8 \%)$ & 0.33 \\
\hline Hypertension & $n=7565,5492(72.6 \%)$ & $n=8349,6223(74.5 \%)$ & 0.70 \\
\hline Peripheral vascular disease & $n=7550,553(7.3 \%)$ & $n=8272,452(5.5 \%)$ & 0.030 \\
\hline Current smoker & $n=7585,2186(28.8 \%)$ & $n=8383,1983(23.7 \%)$ & 0.007 \\
\hline Previous myocardial infarction & $\mathrm{n}=7575,1762(23.3 \%)$ & $\mathrm{n}=8347,1948(23.3 \%)$ & 0.91 \\
\hline Previous percutaneous coronary intervention & $n=7581,2522(33.3 \%)$ & $n=8373,2699(32.2 \%)$ & 0.53 \\
\hline Previous major bleeding or predisposition to bleeding & $\mathrm{n}=7572,48(0.6 \%)$ & $\mathrm{n}=8375,50(0.6 \%)$ & 0.78 \\
\hline
\end{tabular}

Mixed-models $p$ values, accounting for a random effect of hospital identifier.

CAD, coronary artery disease; eGFR, estimated glomerular filtration rate; GLASSY, GLOBAL LEADERS Adjudication Sub-StudY.

$0.61-0.80$ as substantial and $0.81-1.00$ as almost perfect agreement. However, Cohen's suggested interpretation was critiqued as too lenient for health-related studies, because it implies that a score as low as 0.41 might be acceptable. Therefore, we will interpret concordance between IR and CEC endpoints as follows: ${ }^{11}$

$0-0.20$ : none.

0.21-0.39: minimal.

$0.40-0.59$ : weak.

$0.60-0.79$ : moderate

0.80-0.90: strong.

Above 0.90: almost perfect.

\section{ETHICS AND DISSEMINATION}

A complete list is attached in the online supplementary appendix. The study has been registered on ClinicalTrials. Gov, a website that will be also used for reporting of study results and dissemination.

\section{PATIENT AND PUBLIC INVOLVEMENT}

In GLASSY, the research question was developed to complement the investigator assessment. While patients were not directly involved in the design or conception of the study and no specific patient reported outcome has been considered, the extensive characterisation of several non-fatal endpoints is expected to provide a thorough assessment of intervention on patient experience. GLASSY results will be disseminated to patients mainly via the local investigators. We especially take here the opportunity to thank all patients and families who volunteered to help others.

\section{STUDY ORGANISATION}

The European Cardiovascular Research Institute (ECRITrials B.V., Rotterdam, The Netherlands) will act as sponsor of this substudy. The leadership of the GLASSY is composed of the chair (Professor Stephan Windecker) and principal investigator (Professor Marco Valgimigli), in conjunction with the CEC members. Along with the executive committee of the parent study and one representative for each included GLASSY site they will form the publication committee.

\section{DISCUSSION}

CECs are intended to enhance the scientific validity of a clinical trial through systematic, independent and standardised identification, processing and adjudication of suspected events. There are multiple lines of evidence indicating that central and independent adjudication of events may affect the results of a randomised trial by identifying clinically relevant unreported events, ${ }^{12-14}$ by minimising variability and heterogeneity inherently present when several different clinicians and data managers apply definitions of endpoints that are complex and sometimes not well known, ${ }^{15}$ with implications on the interpretation of the effect of a randomised intervention. ${ }^{16}$ Finally, there has been an increasing regulatory emphasis on the requirement of an independent CEC. ${ }^{17-19}$

An analysis of the randomised PURSUIT (Platelet Glycoprotein IIb/IIIa in Unstable Angina: Receptor Suppression Using Integrilin Therapy) trial documented that site investigator and CEC assessments of whether a MI had occurred disagreed in 983 (20\%) of the 5005 patients with suspected MI, mostly reflecting site misclassification 
Table 2 Quality indicators and risk profile of GLOBAL LEADERS patients according to GLASSY inclusion

\begin{tabular}{|c|c|c|c|c|}
\hline \multirow[b]{2}{*}{ No of patients } & \multirow{2}{*}{$\begin{array}{l}\text { GLASSY } \\
\mathrm{n}=7585\end{array}$} & \multirow{2}{*}{$\begin{array}{l}\text { No GLASSY } \\
\mathrm{n}=8383\end{array}$} & \multirow[b]{2}{*}{$P$ value } & \multirow{2}{*}{$\begin{array}{l}\text { Interaction } \\
\text { P value }\end{array}$} \\
\hline & & & & \\
\hline $\begin{array}{l}\text { All-cause mortality or new Q-wave MI or } \\
\text { equivalentLBBBat } 2 \text { years }\end{array}$ & $n=7585,328(4.3 \%)$ & $n=8383,325(3.9 \%)$ & 0.16 & 0.77 \\
\hline All-cause mortality at 2 years & $n=7585,247$ (3.3\%) & $\mathrm{n}=8383,230(2.7 \%)$ & 0.06 & 0.34 \\
\hline New Q-wave MI or equivalent LBBB at 2 years & $n=7585,89(1.2 \%)$ & $\mathrm{n}=8383,97(1.2 \%)$ & 0.93 & 0.34 \\
\hline Primary endpoint complete, $\mathrm{n}(\%)$ & $\mathrm{n}=7585$ & $\mathrm{n}=8383$ & $<0.001$ & 0.75 \\
\hline Complete & $7152(94.3)$ & $7683(91.6)$ & & \\
\hline Vital status unknown & $0(0.0)$ & $8(0.1)$ & & \\
\hline $\begin{array}{l}\text { Patient died post } 2 \text { years and ECG information } \\
\text { unavailable }\end{array}$ & $11(0.1)$ & $16(0.2)$ & & \\
\hline No of protocol deviations/10 patients & $\mathrm{n}=20,0.4(0.1$ to 0.8$)$ & $\mathrm{n}=110,0.6(0.2$ to 1.3$)$ & 0.14 & \\
\hline Statin at discharge & $n=7547,6954(92.1 \%)$ & $n=8324,7747(93.1 \%)$ & 0.78 & \\
\hline $\begin{array}{l}\text { Heart failure or left ventricular ejection fraction } \\
\leq 40 \% \text { treated and ACE or ARB at discharge }\end{array}$ & $\mathrm{n}=251,207(82.5 \%)$ & $\mathrm{n}=284,232(81.7 \%)$ & 0.51 & \\
\hline $\begin{array}{l}\text { Heart failure or left ventricular ejection fraction } \\
\leq 40 \% \text { treated and beta-blockers at discharge }\end{array}$ & $n=157,130(82.8 \%)$ & $n=221,181(81.9 \%)$ & 0.88 & \\
\hline
\end{tabular}

GLASSY includes 20 sites; no GLASSY includes 110 sites; total number of sites was 130.

$P$ values from Mantel-Cox logrank test, interaction $p$ value testing whether the GLASSY versus non-GLASSY sites modify the comparison experimental treatment strategy versus reference treatment strategy for the clinical outcomes. Protocol deviations compared with MannWhitney U-test.

Protocol deviations included: inclusion/exclusion criteria, informed consent procedure, randomisation procedure, study procedures and safety reporting.

ARB, angiotensin receptor blockers; BARC, Bleeding Academic Research Consortium; GLASSY, GLOBAL LEADERS Adjudication Sub-StudY; MI, myocardial infarction; LBBB, left bundle branch block.

of postenrolment MIs (as enrolment MIs) or under-reported periprocedural MIs with a higher mortality associated with CEC-identified MIs as compared with patients with no MI. ${ }^{12}$ Similarly, in post hoc analysis of two large randomised studies testing antithrombotic therapies in patients with coronary artery disease, CEC procedures identified more events (both ischaemia and bleeding) as compared with site investigators. ${ }^{13} 14$ Moreover, independent adjudication of ischaemic and bleeding endpoints may provide important mechanistic information that may deepen understanding of the primary endpoint result of the study by better characterising components of such endpoints including, but not limited to cause of death, subtype of MI according to standardised definitions ${ }^{20}$ and bleeding location.

Also standardised adjudication processes provide the basis for consistency and reproducibility. In large validation effort of all-comer stent trials, a harmonisation process provided a high level of concordance for event adjudication and improved accuracy for final event reporting. ${ }^{15}$

Finally, the presence of a CEC has been strongly advocated by regulatory authorities ${ }^{17}$ and requested in some instances for concern of bias in open-label studies. ${ }^{18}$ Notably, regulatory authorities have been recently involved directly in endpoint definition along with investigators, pharmaceutical and CV device manufacturers and other stakeholders. ${ }^{19}$

GLASSY is a first of its kind scientific study designed to implement CEC processes in the context of a large phase III pragmatic trial intended to collect only IR endpoints. As such, it may provide unique information on how the adoption of CEC processes may affect study results. Some design features of an RCT, including blinding of randomised treatment and independent endpoint adjudication, may be complex, costly and challenging to implement in a pragmatic trial thus limiting study feasibility. However, these characteristics are important to enhance the scientific validity and quality of the evidence generated by minimising detection and/or reporting bias. GLASSY may indirectly allow to assess whether such bias(es) are present in GLOBAL LEADERS by quantifying the concordance (or lack thereof) between IR-adjudicated and CEC-adjudicated endpoints. In other words, to test the value of CECs. This could have relevant implications for the interpretation of the GLOBAL LEADERS results and to inform the design of similar studies in the future. 
Pragmatic clinical trials are fundamental to complement earlier phase studies designed to explore the efficacy of a given intervention. In addition, recent registry-based randomised trials have been appraised owing to their ability to address clinically relevant questions in large representative patient populations at limited cost. Pragmatism, an established concept in clinical research, aims at enhancing generalisability rather than internal validity of a study result and promote clinical or policy decision making by providing evidence for the adoption of a given intervention into real-world clinical practice. ${ }^{21-23}$ To quantify the pragmatism of a clinical trial, tools have been proposed to examine whether key dimensions of a study-such as eligibility, recruitment and primary outcome-are directly related and relevant to usual care. ${ }^{24}$ Importantly, the role of independent endpoint adjudication in this context is a quality rather than a pragmatic issue. If the quality and consistency of endpoint ascertainment can be improved by adjudication without affecting routine patient care, CECs are highly desirable. ${ }^{25}$

A typical strength of CEC processes is to provide standardisation around secondary outcomes or subtype of events, such as characterisation of the modality of death, the location of a bleeding or the type of MI according to the universal Classification, that may be not reliably collected in the absence of standardised definitions and conventions. These data however, are important to fully characterise the efficacy and safety of an antithrombotic treatment intervention, such as that studied in the GLOBAL LEDERS study. According to best adjudication practice, GLASSY will collect and analyse extensive outcome data, beyond the occurrence of the event itself, that were not considered in GLOBAL LEADERS CRF. Additionally, for each non-fatal suspected endpoint, we will assess if the documentation provided by the site was sufficient to understand whether the endpoint has occurred that may allow indirectly estimating the quality of endpoint reporting by the site.

\section{Limitations}

An intrinsic limitation of GLASSY is that GLOBAL LEADERS has been designed as an IR-only study. Therefore, systematic identification of study endpoints is limited by the eCRF and relies on source documentation provided by the site, which reduces the ability to identify all possible potential endpoints.

In addition, GLASSY, for logistical reasons, will be conducted in a representative sample rather than the entire parent study. Although a random sample would have been ideal in this setting, this was neither feasible or financially sustainable. The practical reason to focus enrolment for GLASSY to top enrolling centre may bias the study towards the null hypothesis of no difference between IR-adjudicated and CEC-adjudicated endpoint by selecting best enrolling sites. While this bias is possible, the relatively large study sample $(\approx 50 \%$ of the parent study) makes this possibility unlikely.

\section{CONCLUSIONS}

GLASSY will assess the scientific implications of CEC adjudication processes within a large RCT designed to collect only IR-reported events, to extend the assessment of the effectiveness and safety of the randomised intervention tested in GLOBAL LEADERS to a broad range of non-fatal ischaemic and bleeding endpoints and ultimately to test the value of standardised CEC processess within a pragmatic study design.

\section{Author affiliations}

1University of Pavia, Department of Molecular Medicine, Fondazione IRCCS Policlinico San Matteo, Pavia, Italy

${ }^{2}$ Department of Advanced Biomedical Sciences, Federico II University of Naples, Naples, Italy

${ }^{3}$ Department of Cardiology, Cork University Hospital Group, Cork, Ireland ${ }^{4}$ Department of Cardiology and Critical Care Medicine, Hartcentrum Hasselt, Hasselt, Belgium

${ }^{5}$ Department of Cardiology, Imperial College of London, London, UK

${ }^{6}$ Department of Cardiology, Jessa Hospital, Hasselt, Belgium

${ }^{7}$ Department of Cardiology, Kerckhoff Heart and Thorax Centre, Bad Nauheim, Germany

${ }^{8}$ Department of Cardiology, Imelda Hospital, Bonheiden, Belgium

${ }^{9}$ Department of Cardiology, Fondazione IRCCS Policlinico San Matteo, Pavia, Italy

${ }^{10}$ Center of Cardiovascular Research and Development, American Heart of Poland,

Katowice, Poland

${ }^{11}$ Department of Cardiology, Erasmus MC, Rotterdam, The Netherlands

${ }^{12}$ Department of Cardiology, S. Maria University-Hospital, Terni, Italy

${ }^{13} 3$ rd Med Department, Cardiology and Emergency Medicine, Wilhelminenhospital, Vienna, Austria

${ }^{14}$ Department of Cardiology, OLVG, Amsterdam, The Netherlands

${ }^{15}$ Center for Cardiovascular Research and Development, American Heart of Poland,

Poland, Poland

${ }^{16}$ Department of Cardiology, Azienda Toscana USL Sudest, Arezzo, Italy

${ }^{17}$ Department of Cardiology, Azienda Ospedaliero Universitaria di Ferrara

Arcispedale Sant'Anna, Cona, Italy

${ }^{18}$ Jagiellonian University Medical College, The John Paul II Hospital, Krakow, Poland

${ }^{19}$ Department of Cardiology, Centre Hospitalier Universitaire de Charleroi, Charleroi, Belgium

${ }^{20}$ Department of Cardiology, Ziekenhuis Oost-Limburg, Genk, Belgium

${ }^{21}$ Department of Cardiology, Adzhibadem Siti Klinik Surdechno-sudovi Center, Sofia, Bulgaria

${ }^{22}$ Department of Cardiology, East Lancashire Hospitals NHS Trust, Blackburn, UK

${ }^{23}$ Department of Cardiology and Angiology, Contilia Heart and Vascular Centre, Essen, Germany

${ }^{24}$ Department of Cardiology, PAKS Kozle, Kozle, Poland

${ }^{25}$ Kerckhoff Heart Center, Bad Nauheim, Germany

${ }^{26}$ Assistance Publique-Hôpitaux de Paris, Hôpital Bichat, Paris, France

${ }^{27} \mathrm{CTU}$, Institute of Social and Preventive Medicine and Clinical Trials Unit, Bern, Switzerland

${ }^{28}$ Department of Medicine, Applied Health Research Centre, Li Ka Shing Knowledge Institute of St Michael's Hospital, Toronto, Canada

${ }^{29}$ Department of Cardiology, Inselspital Universitatsspital Bern, Bern, Switzerland

Contributors M Val, SW, SL, AF and EM: study design, manuscript drafting, data interpretation and critical revision. EM, SL, AF and RP: Clinical event committee operations and adjudications. AF: data management. DH and PJ: statistical analysis, data interpretation and critical revision. PV, PS, EB, CL, LJ, MF, AZ, R-JvG, MD, KH, TS, PB, LB, CT, KB, AA, MVro, IP, SG, CN, JP, CH and GS: data acquisition, interpretation and critical revision.

Funding The study is independently financed by resources of a professorship grant at the University of Bern, Switzerland. No extramural funding was used to support this manuscript. The authors are solely responsible for the drafting and editing of the paper and its final contents.

Competing interests SL reports personal fees for advisory board participation from AstraZeneca, Chiesi and The Medicine Company. M Val reports grants from The Medicines Company, grants from Terumo, during the study; grants from 
AstraZeneca, and personal fees from Terumo, St Jude Vascular and Abbott Vascular, outside the submitted work.

Patient consent for publication Not required.

Ethics approval GLASSY has been approved by local ethics committee of all study sites.

Provenance and peer review Not commissioned; externally peer reviewed.

Open access This is an open access article distributed in accordance with the Creative Commons Attribution Non Commercial (CC BY-NC 4.0) license, which permits others to distribute, remix, adapt, build upon this work non-commercially, and license their derivative works on different terms, provided the original work is properly cited, appropriate credit is given, any changes made indicated, and the use is non-commercial. See: http://creativecommons.org/licenses/by-nc/4.0/.

\section{REFERENCES}

1. Valgimigli M, Bueno $\mathrm{H}$, Byrne RA, et al. $2017 \mathrm{ESC}$ focused update on dual antiplatelet therapy in coronary artery disease developed in collaboration with EACTS: The Task Force for dual antiplatelet therapy in coronary artery disease of the European Society of Cardiology (ESC) and of the European Association for CardioThoracic Surgery (EACTS). Eur Heart J 2018;39:213-60.

2. Jakubowski JA, Matsushima N, Asai F, et al. A multiple dose study of prasugrel (CS-747), a novel thienopyridine P2Y12 inhibitor, compared with clopidogrel in healthy humans. Br J Clin Pharmacol 2007;63:421-30.

3. Siller-Matula JM, Trenk D, Schrör K, et al. Response variability to P2Y12 receptor inhibitors: expectations and reality. JACC CardiovasC Interv 2013;6:1111-28.

4. Wallentin L, Becker RC, Budaj A, et al. Ticagrelor versus clopidogrel in patients with acute coronary syndromes. $N$ Engl J Med 2009;361:1045-57.

5. Armstrong PC, Leadbeater PD, Chan MV, et al. In the presence of strong P2Y12 receptor blockade, aspirin provides little additional inhibition of platelet aggregation. J Thromb Haemost 2011;9:552-61.

6. Gargiulo G, Windecker S, Vranckx P, et al. A critical appraisal of aspirin in secondary prevention: is less more? Circulation 2016;134:1881-906.

7. Vranckx P, Valgimigli M, Windecker S, et al. Long-term ticagrelor monotherapy versus standard dual antiplatelet therapy followed by aspirin monotherapy in patients undergoing biolimus-eluting stent implantation: rationale and design of the GLOBAL LEADERS trial. Eurolntervention 2016;12:1239-45.

8. Seltzer JH, Turner JR, Geiger MJ, et al. Centralized adjudication of cardiovascular end points in cardiovascular and noncardiovascular pharmacologic trials: a report from the Cardiac Safety Research Consortium. Am Heart J 2015;169:197-204.

9. Hansson L, Hedner T, Dahlöf B. Prospective randomized open blinded end-point (PROBE) study. A novel design for intervention trials. Prospective Randomized Open Blinded End-Point. Blood Press 1992;1:113-9.

10. Kahan BC, Cro S, Doré CJ, et al. Reducing bias in open-label trials where blinded outcome assessment is not feasible: strategies from two randomised trials. Trials 2014;15:456.

11. McHugh ML. Interrater reliability: the kappa statistic. Biochem Med 2012;22:276-82.

12. Mahaffey KW, Harrington RA, Akkerhuis M, et al. Disagreements between central clinical events committee and site investigator assessments of myocardial infarction endpoints in an international clinical trial: review of the PURSUIT study. Curr Control Trials Cardiovasc Med 2001;2:187-94.

13. Jatene T, Harrington RA, Stone GW, et al. Investigator-Reported Bleeding Versus Post Hoc Adjudication of Bleeding: Lessons From the CHAMPION PHOENIX Trial. J Am Coll Cardiol 2016;67:596-8.

14. Mahaffey KW, Held C, Wojdyla DM, et al. Ticagrelor effects on myocardial infarction and the impact of event adjudication in the PLATO (Platelet Inhibition and Patient Outcomes) trial. J Am Coll Cardiol 2014;63:1493-9.

15. Vranckx P, McFadden E, Cutlip DE, et al. Clinical endpoint adjudication in a contemporary all-comers coronary stent investigation: methodology and external validation. Contemp Clin Trials 2013;34:53-9.

16. Leonardi S, Lopes RD, Steg PG, et al. Implications of different criteria for percutaneous coronary intervention-related myocardial infarction on study results of three large phase III clinical trials: the CHAMPION experience. Eur Heart J Acute Cardiovasc Care 2018;7:pii: 2048872616661692

17. Farb A, Zuckerman BD. Clinical event adjudication in cardiovascular device trials: an food and drug administration perspective. Am Heart J 2017;191:62-4.

18. Lopes RD, Dickerson S, Hafley G, et al. Methodology of a reevaluation of cardiovascular outcomes in the RECORD trial: study design and conduct. Am Heart $J$ 2013;166:208-16. e28.

19. Hicks KA, Mahaffey KW, Mehran R, et al. 2017 Cardiovascular and Stroke Endpoint Definitions for Clinical Trials. J Am Coll Cardiol 2018;71:1021-34.

20. Leonardi S, Truffa AA, Neely ML, et al. A novel approach to systematically implement the universal definition of myocardial infarction: insights from the CHAMPION PLATFORM trial. Heart 2013;99:1282-7.

21. Sacristán JA, Dilla T. Generalizability in Pragmatic Trials. JAMA 2017;317:87-8

22. Fröbert $\mathrm{O}$, Lagerqvist $\mathrm{B}$, Olivecrona GK, et al. Thrombus aspiration during ST-segment elevation myocardial infarction. N Engl J Med 2013;369:1587-97.

23. Lauer MS, D'Agostino RB. The randomized registry trial-the next disruptive technology in clinical research? N Engl $J$ Med 2013;369:1579-81.

24. Loudon K, Treweek S, Sullivan F, et al. The PRECIS-2 tool: designing trials that are fit for purpose. BMJ 2015;350:h2147.

25. Ford I, Norrie J. Pragmatic Trials. N Engl J Med 2016;375:454-63. 